\title{
GnRH-Blocker beeinflusst kardiovaskuläre Erkrankungen nicht
}

_ Die Therapie des fortgeschrittenen Prostatakarzinoms mit LHRH-Agonisten ist mit einem erhöhten Risiko kardiovaskulärer Erkrankungen assoziiert: Innerhalb der ersten vier Monate steigt das Risiko signifikant an und bleibt auch im weiteren Verlauf auf einem signifikant erhöhten Niveau [Keating et al. J Clin Oncol 2006; 24: 4448-56]. Unter dem GnRH-Blocker Firmagon ${ }^{\circledast}$ (Degarelix) dagegen veränderte sich bei Patienten mit fortgeschrittenem Prostatakarzinom (PCa) die Rate an kardiovaskulären Ereignissen (KVE) vor und nach der Behandlung nicht. Zu diesem Ergebnis kommt eine aktuelle Studie mit 1.704 PCaPatienten aus neun prospektiven klinischen Untersuchungen [Smith et al. Journal of Urology, 2011; 186: 1835-42]. In dieser Studie veränderte sich die Rate an kardiovaskulären Ereignissen bei der Gesamtpatientenpopula- tion ein Jahr vor Beginn und während der Degarelix-Therapie von durchschnittlich 22 Monaten nicht (5,5 versus $6,1 / 100$ personyears). In der Subgruppe der Patienten ohne bestehende kardiovaskuläre Erkrankung als Ausgangswert wurde nach Beginn der Degarelix-Therapie eine niedrigere Ereignisrate beobachtet (5,6 versus 4,3/100 Personenjahre, Pj). Dagegen schien sich bei Patienten, die eine kardiovaskuläre Erkrankung im Referenzzeitraum bereits vor der Behandlung hatten, unter Degarelix erwartungsgemäß die Ereignisrate signifikant zu erhöhen (5,3 versus 10,5 Ereignisse pro 100/Pj). Dienten die Ereignisraten während der sechs Monate vor Beginn der Degarelix-Therapie als Referenzzeitraum, wurde keine erhöhte Rate an kardiovaskulären Ereignissen unter dem GnRH-Blocker beobachtet ( 8,9 versus $10,7 / 100 \mathrm{Pj})$. In der Multi- varianzanalyse war eine kardiovaskuläre Erkrankung als Ausgangswert der stärkste unabhängige Prognosefaktor, gefolgt von höherem Alter, Alkoholabstinenz und Adipositas. Weder das Dosisregime noch die Therapiedauer hatten Einfluss auf das Risiko für ein kardiovaskuläres Ereignis. Insgesamt weisen die Ergebnisse von Smith et al. darauf hin, dass Degarelix in keinem ursächlichen Zusammenhang mit kardiovaskulären Ereignissen steht. Möglicherweise ist die entgegengesetzte Wirkung von GnRH-Agonisten und GnRH-Blockern auf die Aktivierung und Proliferation der T-Lymphozyten in arteriosklerotischen Plaques eine Erklärung dafür, warum die verschiedenen Therapieformen mit einem unterschiedlichen Risiko für kardiovaskuläre Erkrankungen assoziiert sind. Werden die T-Lymphozyten aktiviert, könnte dies zum Einreißen arteriosklerotischer Plaques und damit zu thromboembolischen Komplikationen führen.

Nach Informationen von Ferring, Kiel

\section{Inkontinenzversorgung: Wie sieht die Zukunft aus?}

— Die Vertragsbedingungen für die Versorgung von GKV-Patienten mit aufsaugenden Inkontinenzprodukten haben sich in den letzten Jahren deutlich gewandelt. Raimund Koch, Leiter des Referats Gesundheitspolitik der Paul Hartmann AG, stellte die Ergebnisse einer aktuellen Analyse des InkontinenzMarktes im ambulanten Bereich vor. Danach stehen bei den derzeit möglichen Vertragsformen zwischen Kassen und Leistungserbringern an erster Stelle die Beitrittsverträge nach § 127 Abs. 2 SGB V $(57,8 \%)$, gefolgt von den Ausschreibungen nach § 127 Abs. 1 SGB V $(29,5 \%)$ und der Festbetrags-Erstattung (12,7\%).

Kritisch sehen viele Leistungserbringer das Sinken der von den Kassen monatlich pro Patient gezahlten Pauschalen, die derzeit bei den Ausschreibungen teilweise unter 20 Euro liegen. Aus Sicht der Leistungserbringer bezweifelte Uwe Strehlow, Magdeburger Strehlow $\mathrm{GmbH}$, dass die Patienten mit derart niedrigen Pauschalen ausreichend versorgt werden können: „Wir haben uns von den Ausschreibungen verabschiedet, weil aus unserer Sicht damit keine ethische Versorgung möglich ist".

Viele Experten vermuten, dass in naher Zukunft die derzeitigen Vertragsbedingungen durch Festzuschüsse abgelöst werden könnten. „Sind die niedrigen Pauschalen einiger Kassen bereits der Festzuschuss durch die Hintertür?", fragte Koch.

Karin Maag, Mitglied des Bundestages und Berichterstatterin für Heil- und Hilfsmittel der CDU/CSU-Fraktion, machte deutlich, dass vonseiten ihrer Fraktion derzeit keine Veränderungen der gesetzlichen Regelungen angestrebt werden. Als Grund führte sie die kostenmäßige Überschaubarkeit des Hilfsmittelbereichs an. Prof. Hans-Georg Will, Leiter des Referats Heil- und Hilfsmittel im Bundeministerium für Gesundheit, unterstützte diese Position. Es müsse erst dann über Veränderungen nachgedacht werden, wenn die Ausgabenentwicklung dazu Anlass gebe.

Nach Ansicht von Friedrich Stodt, Dezernent Leistungsmanagement der Knappschaft und Klaus Mehring gen Friehoff, Sachgebietsleiter Heilund Hilfsmittelmanagement der Barmer GEK sei dies zu begrüßen. Denn die Kassen müssten die Möglichkeit haben, die derzeitigen Instrumente sinnvoll umzusetzen, wozu eine gewisse Kontinuität vonnöten sei. „Gravierende Änderungen würden nur Unruhe schaffen, und die können wir nicht gebrauchen", so Stodt. Dr. Claudia Bruhn

„Expertenmeeting Inkontinenz-Versorgung: Status und Zukunftsperspektiven",

24. Mai 2012, Berlin

Veranstalter: Paul Hartmann AG, Heidenheim
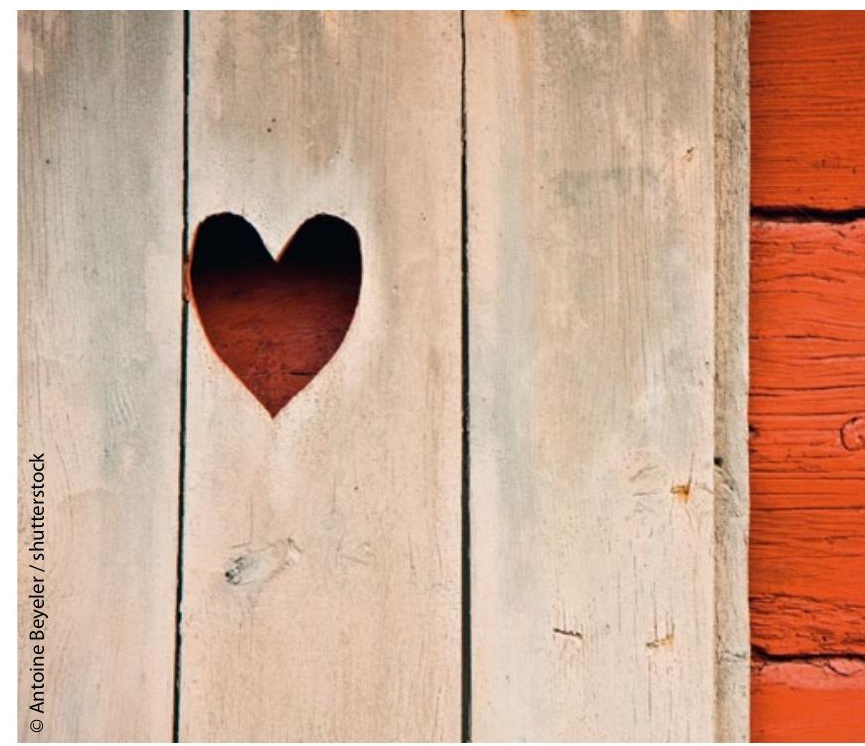